\title{
Cloning and functional validation of early inducible Magnaporthe oryzae responsive CYP76M7 promoter from rice
}

\section{OPEN ACCESS}

Edited by:

Brigitte Mauch-Mani, Université de Neuchâtel, Switzerland

Reviewed by:

Holger Bohlmann,

University of Natural Resources and Life Sciences, Austria

Masamichi Nishiguchi,

Ehime University, Japan

${ }^{*}$ Correspondence: Tilak R. Sharma National Research Centre on Plant Biotechnology, LBS Building, Pusa Campus, New Delhi 110 012, India trsharma@nrcpb.org; trsharma1965@gmail.com

Specialty section: This article was submitted to Plant-Microbe Interaction, a section of the journal Frontiers in Plant Science

Received: 03 February 2015 Accepted: 11 May 2015 Published: 22 May 2015

Citation:

Vijayan J, Devanna BN, Singh NK and Sharma TR (2015) Cloning and functional validation of early inducible Magnaporthe oryzae responsive CYP76M7 promoter from

Front Plant Sci: 6:371. doi: 10.3389/fpls.2015.00371

\section{Joshitha Vijayan, B. N. Devanna, Nagendra K. Singh and Tilak R. Sharma* \\ National Research Centre on Plant Biotechnology, New Delhi, India}

Cloning and functional characterization of plant pathogen inducible promoters is of great significance for their use in the effective management of plant diseases. The rice gene CYP76M7 was up regulated at 24, 48, and 72 hours post inoculation (hpi) with two isolates of Magnaporthe oryzae Mo-ei-11 and Mo-ni-25. In this study, the promoter of CYP76M7 gene was cloned from rice cultivar HR-12, characterized and functionally validated. The Transcription Start Site of CYP76M7 was mapped at 45 bases upstream of the initiation codon. To functionally validate the promoter, $5^{\prime}$ deletion analysis of the promoter sequences was performed and the deletion fragments fused with the $\beta$-glucuronidase (GUS) reporter gene were used for generating stable transgenic Arabidopsis plants as well as for transient expression in rice. The spatial and temporal expression pattern of GUS in transgenic Arabidopsis plants and also in transiently expressed rice leaves revealed that the promoter of CYP76M7 gene was induced by $M$. oryzae. The induction of CYP76M7 promoter was observed at $24 \mathrm{hpi}$ with M. oryzae. We report that, sequences spanning $-222 \mathrm{bp}$ to $-520 \mathrm{bp}$, with the cluster of three W-boxes, two ASF1 motifs and a single GT-1 element may contribute to the $M$. oryzae inducible nature of CYP76M7 promoter. The promoter characterized in this study would be an ideal candidate for the overexpression of defense genes in rice for developing durable blast resistance rice lines.

Keywords: Arabidopsis, CYP76M7, GUS, Magnaporthe, promoter analysis, rice

\section{Introduction}

Plants are being exposed to various biotic and abiotic stresses and they have developed smart developmental and defense mechanisms to counteract these stress conditions. Among biotic stresses, plant pathogens cause widespread damage to the crop plants. The growth and development of organisms is a complex network of different gene products which are controlled by upstream regulatory elements of genes or promoters which induce spatial and temporal specificity to the expression pattern of respective genes. Rice blast disease caused by Magnaporthe oryzae, a hemibiotrophic filamentous plant pathogenic fungus, is one of the most dreaded diseases of rice crop causing an average annual yield loss of $30-50 \%$ in rice growing regions of the world (Sharma et al., 2012).

The $R$ - gene mediated host plant resistance is considered to be one of the most effective, economically feasible and environmental friendly approaches for the effective management of rice 
blast disease. However, host resistance is short lived due to highly variable nature of $M$. oryzae. Therefore, use of multiple $R$ - genes is considered as the most viable and durable option for management of rice blast disease (Hulbert et al., 2001; Das et al., 2012; RoyChowdhury et al., 2012; Devanna et al., 2014). An initial strategy adopted for achieving this was to overexpress resistance genes under the control of constitutive promoters, but in many instances the improved disease resistance was accompanied by reducing growth and associated yield penalty in commercial crops (Hammond-Kosack and Parker, 2003). Therefore the problems associated with constitutive expression can be overcome by using spatially and temporally inducible promoters. There are a number of cloned and characterized promoters which show constitutive, inducible (as pathogen, wound, and chemically induced) and tissue-specific (such as root, stem, pollen, and green tissue-specific) expression (Gurr and Rushton, 2005). Identification and characterization of pathogen inducible promoters would be of larger practical value as they avoid the unnecessary physiological burdens associated with constitutive expression of transgenes on the host plant by restricting their expression to specific time and the site of infection (De Wit, 1992). An ideal pathogen-inducible promoter is the one which is rapidly activated in response to a wide range of invading pathogens and therefore remain effective in providing early and broad-spectrum resistance to the pathogens. In view of this, it is of utmost importance to isolate and characterize a pathogen inducible promoter for the efficient regulation of transgenes and to provide broad spectrum resistance against invading plant pathogens. There are a large number of known pathogen-inducible genes in plants and promoters of a few of those genes have been characterized (Rushton and Somssich, 1998; Gurr and Rushton, 2005). Among the well characterized pathogen inducible promoters, two groups of cis-regulatory elements like GCC-like elements (Ohme-Tagaki et al., 2000) and the W boxes (Rushton et al., 1996; Eulgem et al., 2000) have been widely studied and functionally validated. Secondary metabolites produced during biotic and abiotic stresses are reported to be involved in plant resistance mechanism and therefore the promoters regulating the expression of these genes coding for secondary metabolites are the ideal candidates for the cloning and characterization of novel pathogen inducible promoters.

The CYP76M7 gene (LOC_Os02g36110), a member of P450 monooxygenase, has been reported to be induced after chitin elicitor treatment (Okada et al., 2007). Further, it is known to have a role in the production of antifungal phytocassanes and belongs to a diterpenoid biosynthetic gene cluster located on the rice chromosome 2 (Swaminathan et al., 2009). Previously, phytocassanes have been shown to accumulate in rice during M. oryzae and Rhizoctonia solani infection (Koga et al., 1995). Our microarray based expression study and real time PCR analysis have also confirmed that CYP76M7 gene was highly induced starting from $24 \mathrm{~h}$ after challenged with $M$. oryzae isolate Mo-ei-11 and Mo-ei-25 and was consistently up regulated at both 48 and 72 hours post inoculation (hpi; Vijayan, unpublished data). Recently, in another study, which used RNA sequencing approach for transcriptome analysis at $24 \mathrm{hpi}$ also reported a high level induction of CYP76M7 gene during an incompatible interaction (Bagnaresi et al., 2012). However, the promoter region of this gene has not been characterized at the molecular level. The objectives of the present study were to isolate, characterize and functionally validate a promoter induced by the rice blast fungus M. oryzae.

\section{Materials and Methods}

\section{Plants, Fungal Strains, and Oligos}

Magnaporthe oryzae isolates Mo-ni-25 (Dehradun, Uttarakhand, India) and Mo-ei-11 (Hazaribagh, Jharkhand, India) was collected from the respective locations in India. Seeds of Arabidopsis thaliana L. (Col-0) and indica rice cv. HR-12, susceptible to both Magnaporthe isolates was available at the institute. HR12 was used in the study because the initial microarray experiments, which indicated the early expression of CYP76M7, were performed using rice line HR-12 (Vijayan, unpublished data). The list of oligos used in the present study is given in the Table 1.

\section{Inoculation with $M$. oryzae}

Seedlings of susceptible rice cultivar HR-12 were grown under standard physiological conditions of $16 \mathrm{~h}$ light and $8 \mathrm{~h}$ dark photoperiod at $25 \pm 2{ }^{\circ} \mathrm{C}$. Two-weeks-old rice seedlings were inoculated with two highly virulent and geographical diverse isolates of $M$. oryzae Mo-ni-25 and Mo-ei-11. Conidia of $M$. oryzae isolates were collected from the culture grown on oatmeal agar plates by washing with $0.25 \%$ gelatin and conidial concentration was adjusted to $10^{5}$ spores $\mathrm{ml}^{-1}$ using a haemocytometer. Inoculum was sprayed with an atomizer to create fine droplets of spore suspension, which are retained on the plants. For mock control, plants were inoculated with $0.25 \%$ gelatin only. The experiment was carried out under controlled growth conditions at $25 \pm 2{ }^{\circ} \mathrm{C}$ and $90 \%$ relative humidity.

TABLE 1 | List of oligos used in the present study.

\begin{tabular}{|c|c|}
\hline Primer name & Primer sequence $\left(5^{\prime}-3^{\prime}\right)$ \\
\hline Pcyp2004F & ACAAGCTTAGATCTATGGTTTGTAGGTIIT \\
\hline Pcyp2004R & ACGGATCCGTTCTIITCTCTGGTTCTACCTG \\
\hline DELPcyp1456F & ATAAGCTITGTGTGATGAGCGTCCTTCC \\
\hline DELPcyp1167F & ATAAGCTTTCGGTCGAACACGCATAGC \\
\hline DELPcyp520F & ATAAGCTTCAGCCGTGAGAATCCGTATC \\
\hline DELPcyp222F & ATAAGCTTCGCCATGCAGTAAGGGTATATTC \\
\hline NPTIIF & TGAATGAACTGCATGACGAG \\
\hline NPTIIR & AGCCAACGCTATGTCCTGAT \\
\hline Pcyp GSP1 & GGGATGAACACCATGG \\
\hline Pcyp GSP2 & CGCACGCGCGGAAGGTGTC \\
\hline Pcyp GSP3 & AGGTGGCGGTCGTACTTGG \\
\hline PcypREALF1 & CCAAGTACGACCGCCACCT \\
\hline PcypREALR1 & GCTCGGGATGAACACCATGG \\
\hline 18SRNA-F & CTACGTCCCTGCCCTTTGTACA \\
\hline 18SRNA-R & ACACTTCACCGGACCATTCAA \\
\hline
\end{tabular}




\section{Total RNA Extraction and cDNA Synthesis}

In order to get better results, total RNA was extracted from three different biological replicates of $M$. oryzae and mock inoculated leaf tissues of rice using the Spectrum Plant Total RNA Kit (Sigma). The isolated total RNA was quantified by using Nanodrop quantifier. From each sample, 5000 ng of DNase treated total RNA was used as template for first strand cDNA synthesis. cDNA synthesis was carried out using Protoscript M-MuLV First Strand cDNA Synthesis Kit (Cat. No: E6500S, $\mathrm{NEB}$ ) according to the manufacturer's instructions.

\section{Candidate Promoter Cloning and Quantitative Gene Expression Analysis}

The candidate CYP76M7 gene was selected based on in-house generated microarray experiments data (Vijayan, unpublished data). Further, RT-qPCR was performed to study the expression analysis of CYP76M7 gene (LOC_Os02g36110) using exon specific primers PcypREALF1 and PcypREALR1 (Table 1). The primers were designed using QuantPrime software ${ }^{1}$. cDNA mixture of $2 \mu \mathrm{l}$ was used as a template from each sample. The reaction mixture $20 \mu \mathrm{l}$ was prepared according to the manufacturer's protocol (KAPA Biosystems USA). 18S rRNA primers were used as internal control and PCR was run using Light Cycler 480 II PCR system (Roche Diagnostics, Germany). Each sample was taken as triplicates under following PCR conditions: initial DNA denaturation at $95^{\circ} \mathrm{C}$ for $3 \mathrm{~min}$ followed by 45 cycles of amplification (denaturation at $95^{\circ} \mathrm{C}$ for $3 \mathrm{~s}$; primer annealing at $60^{\circ} \mathrm{C}$ for $20 \mathrm{~s}$ and primer extension at $60^{\circ} \mathrm{C}$ for $1 \mathrm{~s}$ ). The data obtained was normalized with the values of rice $18 \mathrm{~S}$ gene using the $\Delta \Delta \mathrm{C}(\mathrm{T})$ method (Livak and Schmittgen, 2001).

\section{Pathogenesis Study of M. oryzae on Arabidopsis}

In earlier studies it has been shown that M. oryzae causes typical infection on Arabidopsis (Maeda et al., 2009, 2010; Park et al., 2009; Nakao et al., 2011). Hence, for the present study we used A. thaliana L. (Col-0) for genetic transformation and functional characterization of CYP76M7 promoter. Sterilized seeds were sown on Murashige and Skoog (MS; Murashige and Skoog, 1962) medium supplemented with $0.8 \%$ agar and $440 \mathrm{mg} / 1 \mathrm{CaCl}_{2}$. Plated seeds were kept in the dark for 2-3 days at $4^{\circ} \mathrm{C}$ for stratification and subsequently transferred to light and dark regime ( $16 \mathrm{~h}$ light and $8 \mathrm{~h}$ dark) at $22^{\circ} \mathrm{C}$ under $150 \mu \mathrm{mol} \mathrm{m}{ }^{-2}$ $\mathrm{s}^{-1}$ photon flux density and $65 \%$ relative humidity. Four-leaf stage seedlings were later transferred to solarite filled pots and further grown at $22 \pm 2{ }^{\circ} \mathrm{C}$ under long-day conditions in the National Phytotron Facility (NPF). The virulent $M$. oryzae spore suspension (at a concentration of $1 \times 10^{5}$ spores $/ \mathrm{ml}$ ) was used for both spray and spot inoculation of fully expanded healthy rice leaves in three replicates. Both mock and pathogen inoculated plants were grown at $22 \pm 2{ }^{\circ} \mathrm{C}$ and $90 \%$ relative humidity in dark for initial $24 \mathrm{~h}$ and later in light and dark regime of 16 and $8 \mathrm{~h}$, respectively. Phenotypic observations were recorded 3 days post inoculation (dpi).

${ }^{1}$ http://www.quantprime.de/

\section{5' RACE for Mapping TSS}

The Transcription Start Site (TSS) of CYP76M7 transcript was mapped by $5^{\prime}$ RACE (Rapid Amplification of cDNA End) using $5^{\prime}$ First Choice RLM-RACE kit (Ambion, USA). The total RNA isolated from the leaf tissues of pathogen challenged rice plants as described earlier was used as a template for $5^{\prime}$ RACE PCR. RACE PCR was performed according to the manufacturer's protocol. The first strand cDNA synthesis was performed by using GSP1 primer specific to CYP76M7 exon, and in the subsequent steps of PCR based amplification, gene specific primers GSP2 and GSP3 designed from the region upstream to GSP1 were used (Table 1).

\section{Cloning and Analysis of the CYP76M7 Promoter Sequence}

Promoter region of approximately $2.0 \mathrm{~kb}$ was PCR amplified using Pcyp2004F and Pcyp2004R primers specific to CYP76M7 (Table 1) and cloned in pGEM-T Easy cloning vector (Promega Corporation, USA) following the manufacturer's instructions. The clones were confirmed by PCR and restriction enzyme digestion. Three positive clones were custom sequenced (Xcelris Labs Ltd., India) using Sanger's dideoxy method. Each fragment was sequenced twice from both the ends using universal SP6 and T7 specific primers. The sequence reads so obtained were further assembled using Phred/Pharp/Consed Software Package ${ }^{2}$ (Ewing and Green, 1998). The high quality promoter sequence (>30 Phred) assembled was compared using NCBI BLAST with the promoter sequence of the candidate CYP76M7 gene, which was retrieved from the NCBI database using LOC_Os02g36110 sequence as query. The sequence (P0025F02) showing 100\% match with our query sequence was selected and used for in silico analysis. Gene prediction was performed using online gene prediction tool FGENESH software ${ }^{3}$. For in silico analysis of cisregulatory elements in the $2.0 \mathrm{~kb}$ promoter region upstream to the defined TSS we used online software NEW PLACE ${ }^{4}$ (Higo et al., 1999).

\section{Development of CYP76M7 Promoter Deletions and GUS Reporter Gene Fusion Constructs}

High quality consensus CYP76M7 promoter sequence obtained in this study was used for the development of promoter-GUS fusion constructs in plant expression binary vector pBI101, with reporter gene $\beta$-glucuronidase (GUS). For functional validation of the CYP76M7 promoter, $5^{\prime}$ end deletions of the promoter regions were obtained by $\mathrm{PCR}$ amplified fragments. The five sets of forward primers (Pcyp2004F, DELPcyp1456F, DELPcyp1167F, DELPcyp520F, and DELPcyp222F) used for PCR had 5' HindIII restriction enzyme site and were specific to 2030, 1482, 1193, 546 , and 248 bp positions upstream of the TSS of CYP76M7. The common reverse primer (Pcyp2004R) carrying BamHI restriction enzyme site was designed from $5^{\prime}$ UTR region $(+25$ bp; Table 1). Each PCR amplified and purified fragment was digested with HindIII and BamHI restriction enzymes (New England Biolabs,

\footnotetext{
${ }^{2}$ http://www.phrap.org/

${ }^{3}$ www.softberry.com

${ }^{4}$ http://www.dna.affrc.go.jp/PLACE/
} 
UK). Digested products were ligated into the HindIII and BamHI pre-digested pBI101 vector to create promoter::GUS constructs (Supplementary Figure S1). The recombinant clones were confirmed by PCR, restriction digestion analysis using electrophoresis on $1 \%$ agarose gel and sequencing.

\section{In planta Agrobacterium Mediated Transformation of Arabidopsis Plants}

Arabidopsis thaliana (Col-01) plants were grown for 4 weeks at $22 \pm 2{ }^{\circ} \mathrm{C}$ temperature, $18 \mathrm{~h}$ day and $6 \mathrm{~h}$ night conditions at the NPF, New Delhi. Four-weeks-old plants having maximum unopened buds were used for floral dip transformation of different CYP76M7 deletion constructs. This was done by inverting the pot and dipping the unopened buds and leaves of the plants into Agrobacterium suspension prepared in 5\% sucrose with $0.05 \%$ Silwet-L77 solution. The transformed plants were kept at $12 \mathrm{~h}$ in dark and thereafter washed with distilled water. Plants subjected to transformation were then grown to maturity, and then seeds harvested from these plants were sterilized and subjected to selection on kanamycin $(50 \mu \mathrm{g} / \mathrm{ml})$ containing MS medium as described in earlier sections. Overall transformation efficiency of Arabidopsis plants with the different CYP76M7 deletion constructs was found to be between 0.32 and $1.05 \%$ (Table 2). The putative transgenic Arabidopsis plants which grew to four-leaf stage were transferred from the antibiotic containing media plates to solarite containing pots and further grown to maturity to obtain $\mathrm{T}_{2}$ seeds. Transgenic Arabidopsis lines which showed 3: 1 Mendelian segregation ratio was utilized for further experiments (Table 3). Six transgenic homozygous lines each from all the CYP76M7 promoter deletion construct as well as constitutively expressing CaMV $35 \mathrm{~S}$ promoter were selected for functional validation at $\mathrm{T}_{2}$ generation. Transgenic $\mathrm{T}_{2}$ plants were also confirmed for the presence of CYP76M7 promoter and NPTII sequence by PCR based screening. NPTII gene forward primer and CYP76M7 reverse primer were used for PCR amplification and validation.

\section{GUS Histochemical Assay for M. oryzae Responsiveness}

Four-weeks-old healthy and uniformly grown transgenic and control Arabidopsis seedlings were challenged with highly virulent isolates (Moei-11and Mo-ni-25) of M. oryzae. Conidia of $M$. oryzae isolates were collected from cultures grown on

TABLE 2 | Transformation efficiency of Arabidopsis plants using floral dip method.

\begin{tabular}{llll}
\hline $\begin{array}{l}\text { Promoter } \\
\text { Construct } \\
\text { (bp) }\end{array}$ & $\begin{array}{l}\text { Total plants } \\
\text { screened }\end{array}$ & $\begin{array}{l}\text { No. of positive } \\
\text { plants } \\
\text { obtained }\end{array}$ & \% transformation \\
\hline Pcyp2004 & 2836 & 14 & 0.45 \\
Pcyp1456 & 1237 & 6 & 0.48 \\
Pcyp1167 & 2273 & 24 & 1.05 \\
Pcyp520 & 1438 & 5 & 0.34 \\
Pcyp222 & 1832 & 6 & 0.32 \\
\hline
\end{tabular}

${ }^{1}$ T1 seeds of transgenic Arabidopsis were screened on MS medium containing kanamycin antibiotic.
TABLE 3 | The segregation ratio of Knr:Kns populations of transgenic plants.

\begin{tabular}{llll}
\hline Transgenic line & Green seedling* & $\begin{array}{l}\text { Yellow } \\
\text { seedling* }\end{array}$ & $\begin{array}{l}\text { Green/yellow } \\
\text { seedling* }\end{array}$ \\
\hline$(1)$ & 78 & 25 & 3.12 \\
$(2)$ & 132 & 53 & 2.49 \\
$(3)$ & 113 & 40 & 2.82 \\
$(4)$ & 98 & 28 & 3.06 \\
$(5)$ & 81 & 32 & 2.53 \\
$(6)$ & 124 & 38 & 3.26 \\
\hline
\end{tabular}

* Selection on media with kanamycin $(50 \mathrm{mg} / \mathrm{ml})$, the resistant seedling (Knr) are green while the sensitive (Kns) are pale-yellow; the ratio of green/yellow seedling represents the inheritable character of transgenes.

oatmeal agar plates by washing with $0.25 \%$ gelatin, with the conidial concentration adjusted to $10^{5}$ spores $\mathrm{ml}^{-1}$. Plants were spot inoculated with conidia suspension on different parts like leaves, stem and buds. For mock control, plants were inoculated with $0.25 \%$ gelatin only. The experiment was carried out under controlled growth conditions at $22^{\circ} \mathrm{C}$ and $90 \%$ relative humidity. Samples were collected at $24 \mathrm{hpi}$ from inoculated plants and tissues were incubated in GUS assay buffer [containing $1 \mathrm{mM}$ $\mathrm{X}$-gluc (5-bromo-4-chloro-3-indolyl $\beta$-glucuronide) in $50 \mathrm{mM}$ sodium phosphate buffer $[\mathrm{pH} 7.0,1.0 \mathrm{mM}$ potassium ferroand ferri-cyanide, $0.01 \mathrm{M}$ EDTA, $\mathrm{pH}$ 8.0, 0.1\% Triton X100, and $20 \%$ methanol] at $37^{\circ} \mathrm{C}$ for overnight. After overnight incubation, tissues were subjected bleaching using $70 \%$ ethanol for removing chlorophyll. The results were recorded after repeated washing for 4-5 times with fresh $70 \%$ ethanol solution.

\section{GUS Histochemical Assay for Wound and Senescence Responsiveness}

To study the response of CYP76M7 promoter to the wound and senescence, we manually induced wound on the stems as well as leaves of transgenic and control Arabidopsis plants using a razor blade and needle, respectively. Stems and leaves subjected to wounding were assayed for GUS expression at 10 minutes post wounding (mpw). However, for the study of senescenceresponsiveness, we selected those leaves from 6 weeks-old Arabidopsis plants which showed natural senescence and these selected leaves were subjected to GUS histochemical assay. Three replications were used for both wound and senescence responsiveness.

\section{Transient GUS Assay in Rice Leaves}

For transient GUS assay in rice leaves, rice cv. HR-12 was inoculated at two-leaf stage with highly virulent isolate (Moei11) of $M$. oryzae and for mock control, plants were inoculated with $0.25 \%$ gelatin only(as discussed in the previous section). The leaves of both pathogen and mock inoculated plants were collected at 24 hpi and used for particle gun transformation. The leaves were transformed with full length as well as deletion constructs of CYP76M7 promoter::GUS and also with promoterless pBI101 using helium driven Particle Delivery System (PDS 1000, BioRad, USA) using a standard protocol. The 
leaves subjected to bombardment were used for GUS assay at $48 \mathrm{~h}$ post bombardment.

\section{Results}

\section{Expression Analysis of CYP76M7 Gene and Identification of Transcription Start Site}

Differential expression of CYP76M7 gene between $M$. oryzae challenged and mock inoculated susceptible rice plants, HR12 (Supplementary Figure S2) at different time intervals of the experiment were recorded using RT-qPCR (Figure 1A). The CYP76M7 gene was found to be 7.17, 7.39, and 15.96-fold up-regulated in rice leaf tissue after 24, 48, and $72 \mathrm{hpi}$, respectively, with $M$. oryzae isolate Mo-ei-11. Whereas, in case of $M$. oryzae isolate Mo-ni-25, a 6.24, 7.12, and 8.03fold up-regulation was obtained. Increased upregulation of the gene was observed with an increase in the duration of infection and at $72 \mathrm{hpi}$ there is a significant difference $(\sim 7$ fold) in the expression of CYP76M7 between the rice plants inoculated with two M. oryzae isolates, Mo-ei-11 and Mo-ni25. This difference in the fold change can be attributed to the highly virulent nature of Mo-ei-11 in comparison to Mo-ni-25 (Supplementary Figure S2). In order to define position of cisregulatory motifs in the upstream region of CYP76M7 gene, the TSS was determined by using $5^{\prime}$ RACE PCR (Figure 1B). The total RNA isolated from $M$. oryzae challenged rice leaves was used for RACE reaction. Analyses of the sequence obtained from cloned amplicon revealed that the TSS of the gene is defined by the nucleotide 'A' which was found to be located at $45 \mathrm{bp}$ upstream of the translation start codon (ATG) of the CYP76M7 gene.

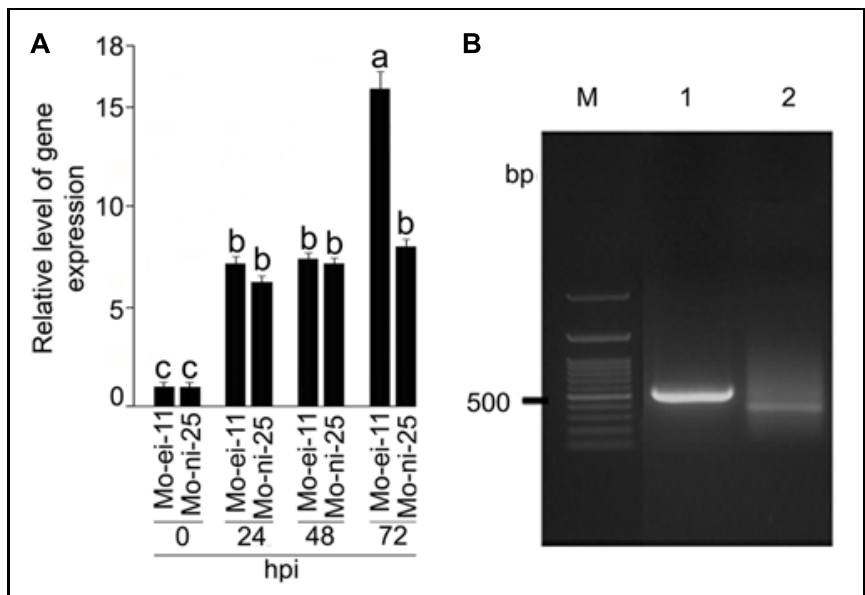

FIGURE 1 | Expression analysis CYP76M7 transcripts and mapping of its Transcription Start Site (TSS). (A) qRT-PCR analysis was performed to analyze the expression of CYP76M7 in response to infection by Magnaporthe oryzae isolate Mo-ei-11 and Mo-ni-25 at 24, 48, and $72 \mathrm{hpi}$. Values represent mean \pm SE. Means with significant deference from each other $(\alpha=0.05)$ is represented with different superscripts according to Tukey's test. (B) 5' RACE (Rapid Amplification of cDNA End) PCR; M: 100 bp GeneRuler (Fermentas) 1: Positive control (provided with the kit), 2: CYP76M7.

\section{Analysis of cis-Regulatory Elements in CYP76M7 Promoter}

The 2,004 bp $5^{\prime}$ regulatory region of the promoter of CYP76M7gene was analyzed for the presence of cis-regulatory elements, found to be associated with pathogen inducible promoters using NEW PLACE database (Figure 2). The important core promoter elements such as TATA Box (CTATAAATAC) at $35 \mathrm{bp}$ upstream to TSS and CAAT box sequences at 92 and $390 \mathrm{bp}$ upstream to TSS were obtained. We also found the presence of motifs like T/G-box (AACGTG) at -66 bp site; while 11 boxes of GT-1 were present at -1848 , $-1829,1681,-1518,-1480,-1,382,-1126,-871,-714$, -603 , and -231 bp positions. An ASF1 motif which is characterized as basic Leucine Zipper (bZIP) protein binding site and reported to be involved in salicylic acid (SA) induced expression of genes (Hwang and Hwang, 2010) was found at $-221,-242$, and -1720 bps. In addition to this, eight ' $W$ ' boxes (TTGAC) containing elements were present at $-1,719$, $-1,420,-753,-669,-621,-493,-250$, and -242 bps positions. A BIHD1OS, the binding site of OsBIHD1, a rice BELL homeodomain transcription factor, was found at $-1292 \mathrm{bp}$.

\section{Establishment of Infection of Arabidopsis with M. oryzae}

The phenotypic response of Arabidopsis (ecotype Col-0) plants inoculated by both spray and spot methods with a spore suspension of $M$. oryzae was observed at $3 \mathrm{dpi}$. At the site of spot inoculation, typical necrotic spots with yellow halo were observed in the case of $M$. oryzae challenged plants, whereas no symptoms were found in mock inoculated plants (Figure 3). This experiment was performed in five replications to establish proper screening protocol. With spray inoculation the pathogen infected leaves developed yellow necrotic patches while no changes were observed in mock inoculated plants.

\section{Validation of the Promoter for Pathogen Responsiveness}

Pathogen responsiveness of CYP76M7 promoter was validated by GUS histochemical assay in stable and transiently transformed Arabidopsis and rice plants, respectively. Independent transgenic Arabidopsis plants with four promoter deletion fragments consisting of Pcyp2004 (2004 bp), Pcyp1456 (1456 bp), Pcyp1167 (1167 bp), and Pcyp520 (520 bp) regions could express GUS in response to pathogen infection at $24 \mathrm{hpi}$, whereas no such induction was observed in the mock inoculated plants (Figure 4A; Supplementary Figure S3A). However, no induction of GUS gene was observed in both pathogen-challenged and mock-inoculated transgenic plants containing Pcyp222 (222 bp) promoter fragment. These findings were further validated by transient expression of GUS in rice leaves. The results obtained in the case of rice were similar to those of Arabidopsis plants. The promoter deletions Pcyp2004, Pcyp1456, Pcyp1167, and Pcyp520 were transiently induced by $M$. oryzae infection (Figure 4B; Supplementary Figure S3B). 

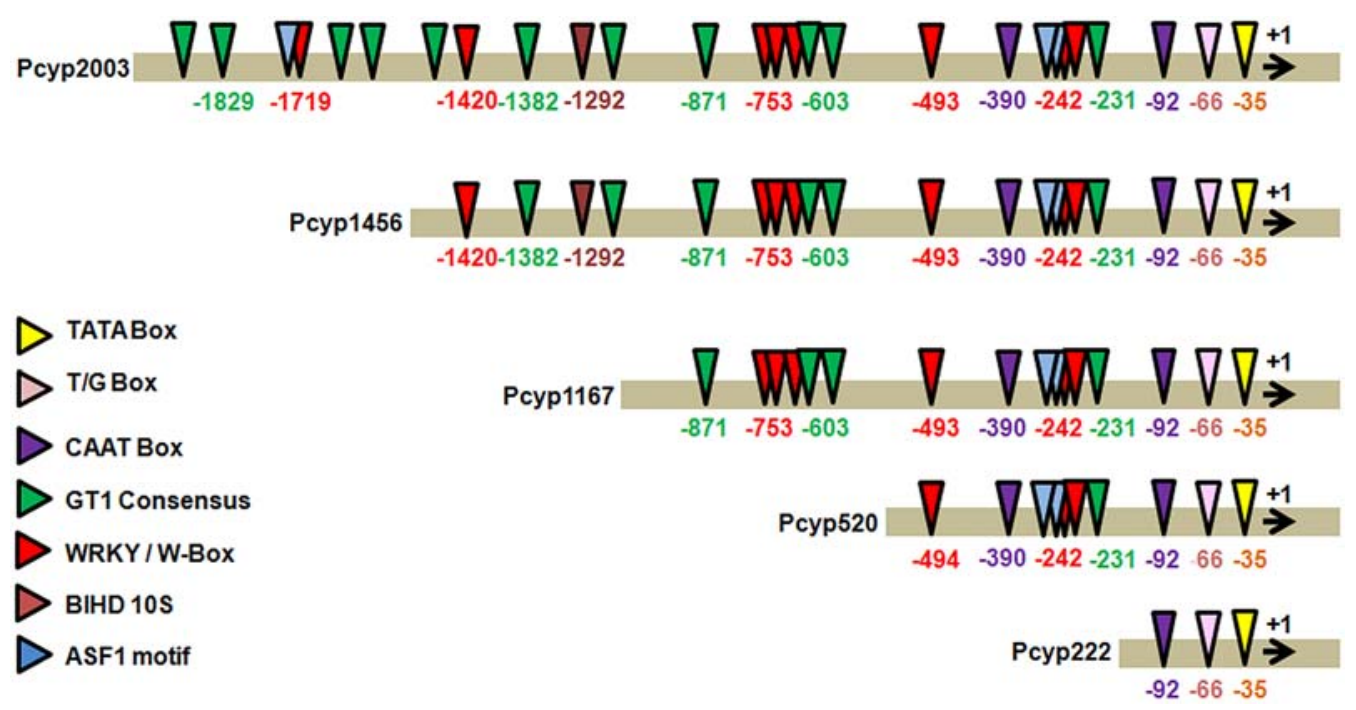

FIGURE 2 | In silico analysis of cis-regulatory elements in candidate gene promoter CYP76M7 (LOC_Os02g36110) and its four deletion fragments using PLACE online tool; Pcyp2004 (CYP76M7 promoter-2004 bp), Pcyp1465 (CYP76M7 promoter-1456 bp), Pcyp1167 (CYP76M7 promoter-1167 bp), Pcyp520 (CYP76M7 promoter-520 bp), and Pcyp222 (CYP76M7 promoter-222 bp).

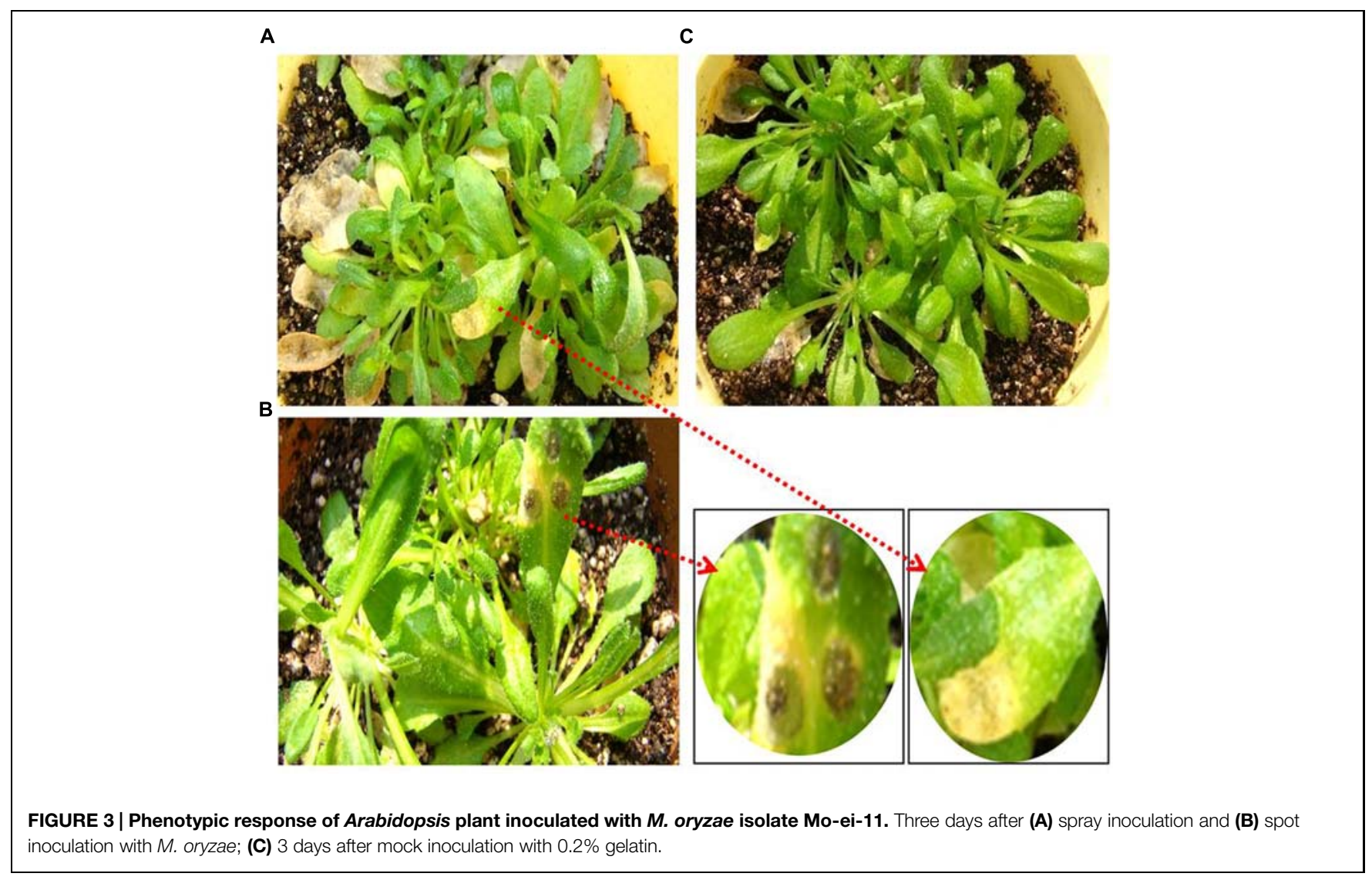

\section{Validation for Wound and Senescence Responsiveness}

The Responsiveness of CYP76M7 promoter to wound as well as senescence was validated by GUS histochemical assay of transformed Arabidopsis plants. The results obtained revealed that transgenic Arabidopsis plants with promoter deletion fragments Pcyp2004, Pcyp1456, Pcyp1167, and Pcyp520 could express GUS in response to pathogen 


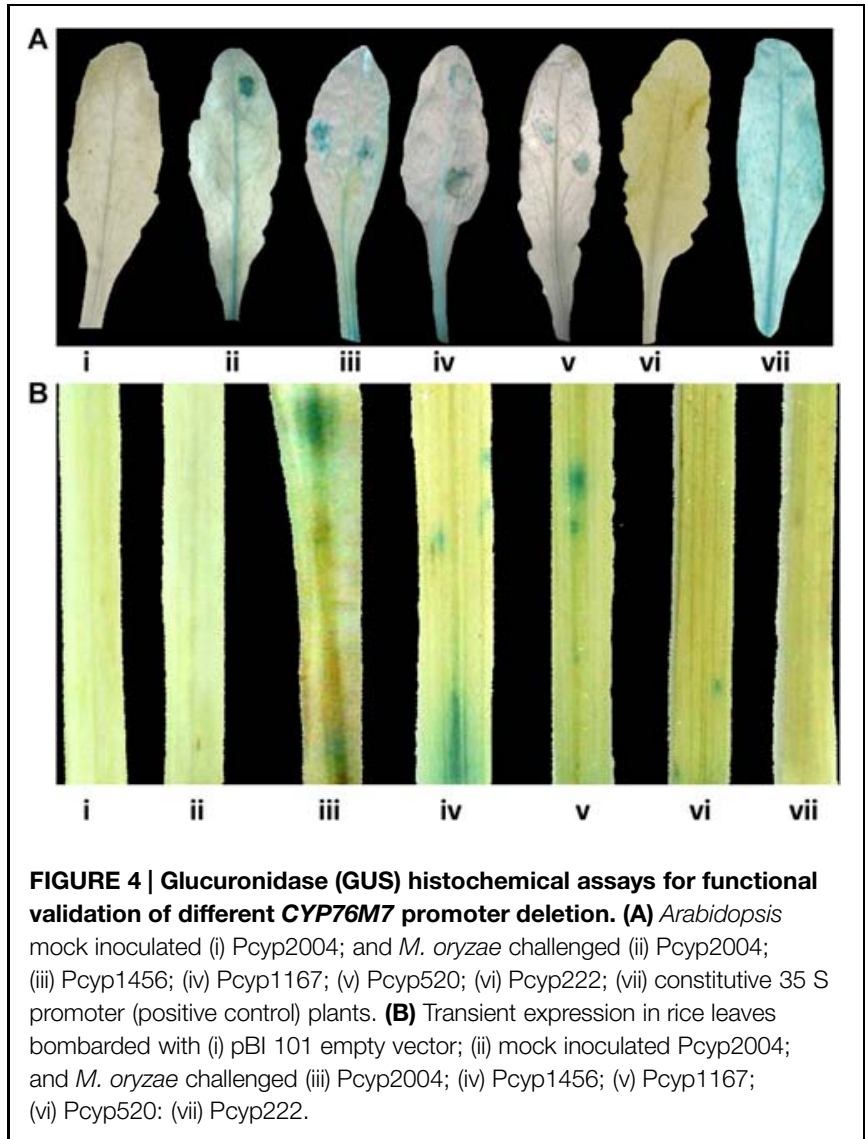

infection at $10 \mathrm{mpw}$ but such expression was limited to cut ends of the stem as well as wounded stem surfaces (Figures 5A,B). Further, the responsiveness of CYP76M7 promoter to senescence revealed that Arabidopsis plants with only full length promoter Pcyp2004 could drive the expression of GUS transcript and no GUS induction was observed in transgenic Arabidopsis plants with deletion fragments Pcyp1456, Pcyp1167, Pcyp520, and Pcyp222 (Figure 5C).

\section{Discussion}

Use of spatially and temporally inducible promoters is considered as an important in obtaining high degree of resistance to plant pathogen. So far only a few plant pathogen-inducible promoters have been well characterized, with respect to their specific patterns and regulation of expression (Gurr and Rushton, 2005). There is a strong need for the identification of early and race-non-specific pathogen-inducible promoters, preferably those which could work across pathogen and species-specific barriers. The tobacco $h s r 203 J$ promoter is an ideal candidate being rapidly induced by more than one pathogen, as early as 9 hpi (Pontier et al., 2001). In the present investigation strong induction of CYP76M7 gene was obtained in the susceptible rice cultivar HR-12 from 24 to 72 hpi in response to infection with geographically diverse isolates of $M$. oryzae. An increased

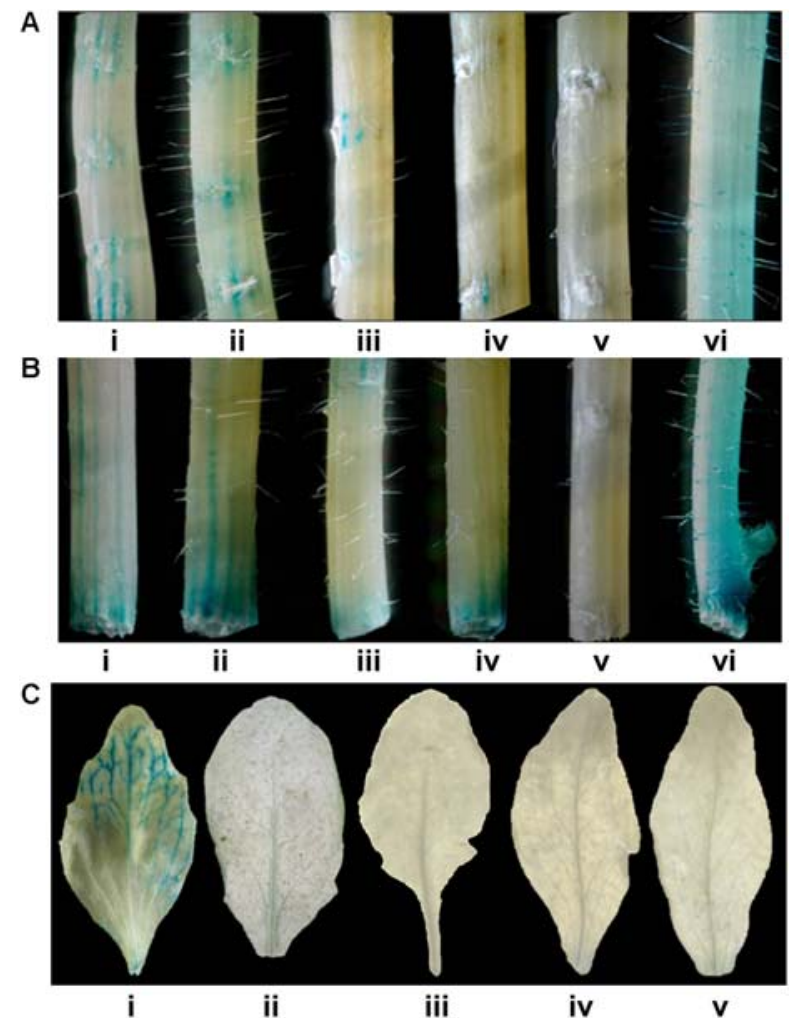

FIGURE 5 | Glucuronidase histochemical assay to know wound responsiveness of CYP76M7 promoter. Stems of different Arabidopsis plants with (A) surface wounding and (B) wounding at the cut ends of (i) Pcyp2004; (ii) Pcyp1456; (iii) Pcyp1167; (vi) Pcyp520: (v) Pcyp222; (vi) 35S promoter. (C) GUS histochemical assay for the leaves undergoing senescence in different transgenic Arabidopsis plants containing CYP76M7 deletions; (i) Pcyp2004; (ii) Pcyp1456; (iii) Pcyp1167; (iv) Pcyp520: (v) Pcyp222.

upregulation of the gene was observed with an increase in the duration of infection and at 72 hpi there is a significant difference $(\sim 7$-fold) in the expression of CYP76M7 between the rice plants inoculated with two $M$. oryzae isolates, Mo-ei11, and Mo-ni-25. The sevenfold difference in the expression pattern of CYP76M7 could be attributed to the highly virulent nature of Mo-ei-11 in comparison to Mo-ni-25. Similar results of $8.05,8.08$, and 10.45-fold change were obtained at 24, 48, and $72 \mathrm{hpi}$, respectively, for CYP76M7 gene using microarray experiments (Unpublished data). Thus, the expression pattern of the CYP76M7 correlates well by both the approaches. Previous study has also reported the induction of CYP76M7 in resistant and susceptible rice genotypes (Bagnaresi et al., 2012).

In the present study, nucleotide sequence analysis of CYP76M7 promoter from O. sativa $\mathrm{cv}$. HR12 showed the presence of TATA box, CAAT box, T/G, GT-1, and ASF1 motifs, as well as WRKY TF and BIHD1OS binding sites. TATA box is necessary for binding of general transcription factors, whereas CAAT box is responsible for the tissue specific promoter activity (Shirsat et al., 1989). T/G-box (AACGTG) has the binding site of 
basic region/helix-loop-helix (bHLH) proteins and is known to be involved in Jasmonic Acid (JA) induction (Boter et al., 2004). The GT-1 motif (GAAAAA) is found to have a role in pathogen and salt-induced gene expression in soybean (Park et al., 2004). The ASF1 motif which is characterized as bZIP protein binding site has been reported to be involved in SA induced expression of the genes (Hwang and Hwang, 2010). Presence of eight W-boxes (TTGAC) containing elements, which are known to bind the WRKY family of transcription factors (Rushton and Somssich, 1998; Nischiuchi et al., 2004) involved in SA -induced expression signifies their role in responsiveness of CYP76M7 promoter to M. oryzae infection. The W-box elements found in the CYP76M7 promoter were found to have a WRKY71OS TF binding site and these elements are reported to be induced by rice blast fungus M. oryzae (Kim et al., 2000; Liu et al., 2006). BIHD1OS sites are the binding site of OsBIHD1, rice BELL homeodomain transcription found in disease resistance response genes (Luo et al., 2005).

Previously, the pathogenecity of M. oryzae on Arabidopsis has been reported (Park et al., 2009). Our assumption in carrying out the functional validation of CYP76M7 promoter in Arabidopsis were the following (i) to determine if, the promoter would be functional across monocot-dicot barrier and (ii) to understand the nuances of pathogen-responsive expression in a non-host model plant system.

The PmPR10-1.13 promoter from Pinus monticola, induced by Cronartium ribicola was reported to be directing the enhanced expression of GUS expression following pathogen infection and by wounding treatment in case of transgenic Arabidopsis plants (Liu et al., 2005). Similarly, promoter of the soybean GmaPPO12 gene was found to be rapidly and strongly induced by Phytophthora sojae infection and induced GUS reporter gene expression after infection in both transient expression assays in Nicotiana benthamiana and stable transgenic soybean hairy roots (Chai et al., 2013). In the present study, 5' deletions of CYP76M7 promoter were fused with promoter less GUS reporter gene and its expression was analyzed in stable and transiently expressed Arabidopsis and rice systems, respectively. In Arabidopsis, the CYP76M7 promoter-directed the expression of GUS gene upon pathogen infection and also by wounding treatment and senescence, both of which generally mimic the endogenous expression pattern of pathogen responsive genes (Liu et al., 2005). Further the transient expression analysis in tomato reports that wounding induces several resistance genes (PINIIb, PR1b, PR5, PR7, and peroxidase) locally and/or systemically and ethylene perception is needed for the full scale induction of all these genes, except peroxidase (Francia et al., 2008). Our study indicates that the promoter which is responsive to pathogen might also be induced by wounding. But, the GUS expression in Arabidopsis was restricted to only stem and petioles and no expression was observed in wounded leaves. Our study also reveals that the promoter region spanning -222 to -520 bp confers $M$. oryzae as well as wound inducibility to CYP76M7 promoter, which may be attributed to the presence of a cluster of functional motifs like three W-boxes, two ASF1 motifs and one GT1 element in the corresponding region. These motifs have been functionally validated and are known to impart stress inducible response to the respective promoters (Rushton and Somssich, 1998; Nischiuchi et al., 2004; Park et al., 2004; Zhang et al., 2007). On the contrary, the response of CYP76M7 to senescence revealed that only full length promoter could induce the expression of GUS gene. This may be attributed to the presence of the ASF-1 motif at -1720 position. Previously, it has been shown that antisilencing function 1a (ASF1a), plays an important role in the formation of SAHF, which contributes to the irreversible exit from the cell cycle in the cells undergoing senescence by inhibiting the expression of cell proliferation-promoting genes (Zhang et al., 2007).

In this study, we have cloned, characterized and functionally validated the CYP76M7 gene promoter for its induction during M. oryzae infection in Arabidopsis and rice. The pathogenicity of M. oryzae on Arabidopsis was confirmed multiple times. GUS histochemical assay analysis of stable transformed Arabidopsis as well as the transient transformed rice leaves revealed that the promoter of CYP76M7 is induced by M. oryzae. Therefore, we report that the cluster of two W-boxes, two ASF1 motifs and a single GT-1 element present between -222 to $-520 \mathrm{bp}$ may contribute to the $M$. oryzae inducible nature of CYP76M7 promoter. Therefore the promoter cloned and functionally validated in this study would be an ideal candidate for a $M$. oryzae inducible promoter.

\section{Author Contributions}

TRS: conceived and designed the experiments. JV and DN: performed the experiments and analyzed the data. TRS, JV, and $\mathrm{DN}$ : wrote the paper.

\section{Acknowledgments}

The financial assistance received by TRS from National Agricultural Innovation Project (NAIP; C4/C1071), Indian Council of Agricultural Research, is gratefully acknowledged. TRS is also thankful to the head of the National Phytotron Facility for providing space for plant growth and maintenance. We thank Dr. M. Variar and Dr. U. D. Singh for providing $M$. oryzae isolates from their respective regions.

\section{Supplementary Material}

The Supplementary Material for this article can be found online at: http://journal.frontiersin.org/article/10.3389/fpls.2015.00371 


\section{References}

Bagnaresi, P., Biselli, C., Orru, L., Urso, S., Crispino, L., Abbruscato, P., et al. (2012). Comparative transcriptome profiling of the early response to Magnaporthe oryzae in durable resistant vs susceptible rice (Oryza sativa L.) genotypes. PLoS ONE 7:e51609. doi: 10.1371/journal.pone. 0051609

Boter, M., Ruiz-Rivero, O., Abdeen, A., and Prat, S. (2004). Conserved MYC transcription factors play a key role in jasmonate signaling both in tomato and Arabidopsis. Genes Dev. 18, 1577-1591. doi: 10.1101/gad. 297704

Chai, C., Lin, Y., Shen, D., Wu, Y., Li, H., and Dou, D. (2013). Identification and functional characterization of the soybean gmappo12 promoter conferring Phytophthora sojae induced expression. PLoS ONE 8:e67670. doi: 10.1371/journal.pone.0067670

Das, A., Soubam, D., Singh, P. K., Thakur, S., Singh, N. K., and Sharma, T. R. (2012). A novel blast resistance gene, Pi54rh cloned from wild species of rice, Oryza rhizomatis confers broad spectrum resistance to Magnaporthe oryzae. Funct. Integr. Genomics 12, 215-228. doi: 10.1007/s10142-0120284-1

De Wit, P. J. G. M. (1992). Molecular characterisation of gene-for-gene systems in plant-fungus interactions and the application of avirulence genes in control of plant pathogens. Annu. Rev. Phytopathol. 30, 391-418. doi: 10.1146/annurev.py.30.090192.002135

Devanna, N. B., Vijayan, J., and Sharma, T. R. (2014). The blast resistance gene Pi54of cloned from Oryza officinalis interacts with Avr-Pi54 through its novel non-LRR domains. PLoS ONE 9:e104840. doi: 10.1371/journal.pone. 0104840

Eulgem, T., Rushton, P. J., Robatzek, S., and Somssich, I. E. (2000). The WRKY superfamily of plant transcription factors. Trends Plant Sci. 5, 199-206. doi: 10.1016/S1360-1385(00)01600-9

Ewing, B., and Green, P. (1998). Base calling sequencer traces using Phred II. Error probabilities. Genome Res. 8, 186-194. doi: 10.1101/gr.8. 3.175

Francia, D., Demaria, D., Calderini, O., Ferraris, L., Valentino, D., Arcioni, S., et al. (2008). Do pathogen-specific defense mechanisms contribute to wound-induced resistance in tomato? Plant Signal. Behav. 3, 340-341. doi: 10.4161/psb.3.5.5351

Gurr, S. J., and Rushton, P. J. (2005). Engineering plants with increased disease resistance: how are we going to express it? TRENDS Biotech. 23, 283-290. doi: 10.1016/j.tibtech.2005.04.009

Hammond-Kosack, K. E., and Parker, J. E. (2003). Deciphering plant pathogen communication: fresh perspectives for molecular resistance breeding. Curr. Opin. Biotechnol. 14, 77-193. doi: 10.1016/S0958-1669(03) 00035-1

Higo, K., Ugawa, Y., Iwamoto, M., and Korenaga, T. (1999). Plant cis-acting regulatory DNA elements (PLACE) database. Nucl. Acids Res. 27, 297-300. doi: 10.1093/nar/27.1.297

Hulbert, S. H., Webb, C. A., Smith, S. M., and Sun, Q. (2001). Resistance gene complexes: evolution and utilization. Annu. Rev. Phytopathol 39, 285-312. doi: 10.1146/annurev.phyto.39.1.285

Hwang, S. H., and Hwang, D. J. (2010). Isolation and characterization of rice NPR1 promoter. Plant Biotechnol. Rep. 4, 29-35. doi: 10.1007/s11816-0090116-5

Kim, C. Y., Lee, S. H., Park, H. C., Bae, C. G., Cheong, Y. H., Choi, Y. J., et al. (2000). Identification of rice blast fungal elicitor-responsive genes by differential display analysis. Mol. Plant Microbe. In. 13, 470-474. doi: 10.1094/MPMI.2000. 13.4.470

Koga, J., Shimura, M., Oshima, K., Ogawa, N., Yamauchi, T., and Ogasawara, N. (1995). Phytocassanes A, B, C, and D, novel diterpene phytoalexins from rice, Oryza sativa L. Tetrahedron 51, 7907-7918. doi: 10.1016/0040-4020(95) 00423-6

Liu, J. J., Ekramoddoullah, A. K., Piggott, N., and Zamani, A. (2005). Molecular cloning of a pathogen/wound inducible PR10 promoter from Pinus monticola and characterization in transgenic Arabidopsis plants. Planta 221, 159-169. doi: $10.1007 /$ s00425-004-1428-x
Liu, X., Bai, X., Wang, X., and Chu, C. (2006). OsWRKY71, a rice transcription factor, is involved in rice defense response. J. Plant Physiol. 164, 969-979. doi: 10.1016/j.jplph.2006.07.006

Livak, K. J., and Schmittgen, T. D. (2001). Analysis of relative gene expression data using real-time quantitative PCR and the 2(-Delta Delta C(T) method. Methods 25, 402-408. doi: 10.1006/meth.2001.1262

Luo, H., Song, F., Goodman, R. M., and Zheng, Z. (2005). Up-regulation of OsBIHD1, a rice gene encoding BELL homeodomain transcriptional factor, in disease resistance responses. Plant Biol. 7, 459-468. doi: 10.1055/s-2005865851

Maeda, K., Houjyou, Y., Komatsu, T., Hori, H., Kodaira, T., and Ishikawa, A. (2009). AGB1 and PMR5 contribute to PEN2-mediated preinvasion resistance to Magnaporthe oryzae in Arabidopsis thaliana. Mol. Plant Microbe. Interact. 22, 1331-1340. doi: 10.1094/MPMI-2211-1331

Maeda, K., Houjyou, Y., Komatsu, T., Hori, H., Kodaira, T., and Ishikawa, A. (2010). Nonhost resistance to Magnaporthe oryzae in Arabidopsis thaliana. Plant Signal. Behav. 5, 755-756. doi: 10.4161/psb.5.6. 11770

Murashige, T., and Skoog, F. (1962). A revised medium for rapid growth and bioassays with tobacco cultures. Physiol. Plant. 15, 473-497. doi: 10.1111/j.13993054.1962.tb08052.x

Nakao, M., Nakamura, R., Kita, K., Inukai, R., and Ishikawa, A. (2011). Non-host resistance to penetration and hyphal growth of Magnaporthe oryzae in Arabidopsis. Sci. Rep. doi: 10.1038/ srep00171

Nischiuchi, T., Shinshi, H., and Suzuki, K. (2004). Rapid and transient activation of transcription of the ERF3 gene by wounding in tobacco leaves - Possible involvement of NtWRKYs and autorepression. J. Biol. Chem. 279, 5535555361. doi: 10.1074/jbc.M409674200

Ohme-Tagaki, M., Suzuki, K., and Shinshi, H. (2000). Regulation of ethyleneinduced transcription of defense genes. Plant Cell Physiol. 41, 1187-1192. doi: 10.1093/pcp/pcd057

Okada, A., Shimizu, T., Okada, K., Kuzuyama, T., Koga, J., Shibuya, N., et al. (2007). Elicitor induced activation of the methylerythritol phosphate pathway towards phytoalexin biosynthesis in rice. Plant Mol. Biol. 65, 177-187. doi: 10.1007/s11103-007-9207-2

Park, H. C., Kim, M. L., Kang, Y. H., Jeon, J. M., Yoo, J. H., Kim, M. C., et al. (2004). Pathogen- and $\mathrm{NaCl}$ induced expression of the SCaM-4 promoter is mediated in part by a GT-1 box that interacts with a GT-1like transcription factor. Plant Physiol. 135, 2150-2161. doi: 10.1104/pp.104. 041442

Park, J. Y., Jin, J., Lee, Y. W., Kang, S., and Lee, Y. H. (2009). Rice blast fungus (Magnaporthe oryzae) infects Arabidopsis via a mechanism distinct from that required for the infection of rice. Plant Physiol. 149, 474-486. doi: $10.1104 /$ pp. 108.129536

Pontier, D., Balague, C., Bezombes-Marion, I., Tronchet, M., Deslandes, L., and Roby, D. (2001). Identification of a novel pathogen-responsive element in the promoter of the tobacco gene HSR203J, a molecular marker of the hypersensitive response. Plant J. 26, 495-507. doi: 10.1046/j.1365-313x.2001. 01049.x

RoyChowdhury, M., Jia, Y. L., Jackson, A., Jia, M. H., Fjellstrom, R., and Cartwright, R. D. (2012). Analysis of rice blast resistance gene Pi-z in rice germplasm using pathogenicity assays and DNA markers. Euphytica 184, 35-46. doi: 10.1007/s10681-011-0481-3

Rushton, P. J., and Somssich, I. E. (1998). Transcriptional control of plant genes responsive to pathogens. Curr. Opin. Plant Biol. 1, 311-315. doi: 10.1016/13695266(88)80052-9

Rushton, P. J., Torres, J. T., Parniske, M., Wernert, P., Hahlbrock, K., and Somssich, I. E. (1996). Interaction of elicitor-induced DNA binding proteins with elicitor response elements in the promoters of parsley PR1 genes. EMBO J. 15, 56905700 .

Sharma, T. R., Rai, A. K., Gupta, S. K., Vijayan, J., Devanna, B. N., and Ray, S. (2012). Rice blast management through host resistance: retrospect and prospects. Agric. Res. 1, 37-52. doi: 10.1007/s40003-0110003-5 
Shirsat, A., Wilford, N., Croy, R., and Boulter, D. (1989). Sequences responsible for the tissue specific promoter activity of a pea legumin gene in tobacco. Mol. Gen. Genet. 215, 326-331. doi: 10.1007/BF00339737

Swaminathan, S., Moron, D., Wang, Q., Fulton, D. B., and Peters, R. J. (2009). CYP76M7 Is an ent-cassadiene C11a-hydroxylase defining a second multifunctional diterpenoid biosynthetic gene cluster in rice. Plant Cell 21, 3315-3325. doi: $10.1105 /$ tpc. 108.063677

Zhang, R., Chen, W., and Adams, P. D. (2007). Molecular dissection of formation of senescence-associated heterochromatin foci. Mol. Cell Biol. 27, 2343-2358. doi: 10.1128/MCB.02019-06
Conflict of Interest Statement: The authors declare that the research was conducted in the absence of any commercial or financial relationships that could be construed as a potential conflict of interest.

Copyright (c) 2015 Vijayan, Devanna, Singh and Sharma. This is an open-access article distributed under the terms of the Creative Commons Attribution License (CC BY). The use, distribution or reproduction in other forums is permitted, provided the original author(s) or licensor are credited and that the original publication in this journal is cited, in accordance with accepted academic practice. No use, distribution or reproduction is permitted which does not comply with these terms. 